\title{
An anecdotal report by an Oxford basic neuroscientist: effects of acetyl-DL-leucine on cognitive function and mobility in the elderly
}

Frances Platt $\mathrm{PhD}^{1}$ and Michael Strupp ${ }^{2}, \mathrm{MD}$

${ }^{1}$ Department of Pharmacology, University of Oxford, UK

${ }^{2}$ Department of Neurology and German Center for Vertigo and Balance Disorders, University Hospital Munich, Munich, Germany

F.P.'s father is a 90-year-old retired physician who has experienced progressive problems with balance over the past five years. He also experienced incomplete arousal wakefulness over the past two years. He had polio in his twenties and has aspects of post-polio syndrome, including partial vocal cord paralysis, muscle weakness and spinal misalignment, primarily neck and upper back. He leads an independent active life, reads prolifically and uses a laptop computer to communicate with family. His only medication was glaucoma eye drops at the time of this study.

To determine if any of his clinical signs would respond to acetyl-DLleucine (Tanganil ${ }^{\mathrm{TM}}$ ) he took a one-month course comprising nine $500 \mathrm{mg}$ capsules taken TID with meals. By 7-10 days of treatment he was more confident in his walking due to improved balance. In a home setting "get up and go test" he was consistently $25 \%$ faster than pre-treatment assessments. He had recently been struggling to get in and out of bed, but that resolved at about the same time.

At approximately 2-3 weeks of treatment he suddenly described being fully alert on waking and that his mind had cleared. His family noticed a significant improvement in his cognitive function, spatial awareness and general demeanor. He experienced no side effects.

After one month he ceased taking acetyl-DL-leucine. His balance remained good for two weeks off medication then deteriorated, where as his cognitive changes gradually declined back to his pre-treatment status over one month. The "get up and go" test was also similar to his pre-treatment performance level.

He therefore initiated another month of treatment and found that the improvements he had noticed in the first month of treatment rapidly returned and he now remains on acetyl-DL-leucine at a dose of nine tablets a day taken TID with meals.

Despite his initial skepticism, he and his family agree that the acetylDL leucine treatment resulted in significant benefit on multiple neurological systems, in particular mobility and cognitive function, greatly improving his quality of life.

Acetyl-DL-leucine has been used in France to treat acute vertiginous symptoms since 1957. Despite a number of proposed hypotheses, including a stabilization of membrane potential, its pharmacological and electrophysiological modes of action remain unclear (1;2). An FDG- $\mu$ PET 
study in a rat model of an acute unilateral labyrinthectomy (3) showed a significant effect of an L-enantiomer $\mathrm{N}$-acetyl-L-leucine on postural compensation by activation of the vestibulo-cerebellum and a deactivation of the posterolateral thalamus (4). The improvement of cerebellar symptoms in humans in a case series with cerebellar patients of different etiologies (5) indicated the therapeutic efficacy of acetyl-DL-leucine. Another case series did not find benefit (6). In a third recent case series in 12 patients with Niemann-Pick type $\mathrm{C}$ this agent caused significant improvement of ataxia (7). Interestingly, the parents of three out of these 12 patients also described remarkable behavioral improvement in terms of affect stabilization, improvement of cooperation and ability to act independently in daily life. This is in line with this short report. Further, a PET study in patients with ataxia of different etiologies given acetyl-DL-leucine demonstrated an increased metabolism in the midbrain and lower brainstem in responders (8), which could explain the benefit on the patient's mobility.

Taken together, this anecdotal report - with its limitations - suggests the potential for acetyl-DL-leucine to be of benefit in the elderly, not only in terms of mobility but also of cognitive function. An observational study in more subjects with impaired cognitive function and mobility using quantitative outcome measures is therefore warranted, in particular since we currently cannot offer many therapeutic options to patients with these very frequent signs and symptoms of aging.

The authors declare no conflicts of interest. 


\section{Reference List}

(1) Vibert N, Vidal PP. In vitro effects of acetyl-DL-leucine (tanganil) on central vestibular neurons and vestibulo-ocular networks of the guinea-pig. Eur J Neurosci 2001 February;13(4):735-48.

(2) Ferber-Viart C, Dubreuil C, Vidal PP. Effects of acetyl-DL-leucine in vestibular patients: a clinical study following neurotomy and labyrinthectomy. Audiol Neurootol 2009;14(1):17-25.

(3) Zwergal A, Schlichtiger J, Xiong G, Beck R, Gunther L, Schniepp R et al. Sequential [F]FDG microPET whole-brain imaging of central vestibular compensation: a model of deafferentation-induced brain plasticity. Brain Struct Funct 2014 October 1 .

(4) Gunther L, Beck R, Xiong G, Potschka H, Jahn K, Bartenstein P et al. N-acetyl-Lleucine accelerates vestibular compensation after unilateral labyrinthectomy by action in the cerebellum and thalamus. PLoS One 2015;10(3):e0120891.

(5) Strupp M, Teufel J, Habs M, Feuerecker R, Muth C, van de Warrenburg BP et al. Effects of acetyl-DL-leucine in patients with cerebellar ataxia: a case series. J Neurol 2013 October;260(10):2556-61.

(6) Pelz JO, Fricke C, Saur D, Classen J. Failure to confirm benefit of acetyl-DL-leucine in degenerative cerebellar ataxia: a case series. J Neurol 2015 May;262(5):13735.

(7) Bremova T, Malinova V, Amraoui Y, Mengel E, Reinke J, Kolnikova M et al. Acetyldl-leucine in Niemann-Pick type C: A case series. Neurology 2015 October 20;85(16):1368-75.

(8) Becker-Bense S, Feuerecker, R, Xiong G, Feil K, Bartenstein P, Strupp M, Dieterich M. FDG-PET in patients with cerebellar ataxia on- vs. off-treatment with AcetylDL-leucine. Abstract EAN 2015. 